\title{
Structural Health Monitoring of Civil Structures: New Methodologies and Field Applications 2012
}

\author{
Piervincenzo Rizzo, ${ }^{1}$ Lingyu (Lucy) Yu, ${ }^{2}$ and Alessandro Marzani ${ }^{3}$ \\ ${ }^{1}$ Department of Civil \& Environmental Engineering, SWANSON School of Engineering, University of Pittsburgh, \\ Pittsburgh, PA 15261, USA \\ ${ }^{2}$ Laboratory for Active Materials and Smart Structures, Department of Mechanical Engineering, \\ University of South Carolina, Columbia, SC 29908, USA \\ ${ }^{3}$ Department of Civil, Environmental and Materials Engineering, University of Bologna, 40126 Bologna, Italy
}

Correspondence should be addressed to Piervincenzo Rizzo, pir3@pitt.edu

Received 12 February 2012; Accepted 12 February 2012

Copyright ( 2012 Piervincenzo Rizzo et al. This is an open access article distributed under the Creative Commons Attribution License, which permits unrestricted use, distribution, and reproduction in any medium, provided the original work is properly cited.

This special issue continues the streamline of papers in Advances in Civil Engineering on the structural health monitoring (SHM) of civil infrastructures. The streamline was initiated in 2010 with the publication of the special issue titled Structural Health Monitoring for Civil Structures: From the Lab to the Field.

This 2012 edition collects five research original papers that mainly cover the monitoring of bridges and pipeline embracing a variety of SHM methodologies including acoustic emission, guided ultrasonic waves, imaging, vibration measurements, and system identification. A brief description of each contribution is provided below.

A team of researchers from the University of South Carolina (USA) and MISTRAS Group (USA) presented the use of passive acoustic emission piezoelectric wafer active sensors (PWAS) to monitor fatigue cracking in steel bridges. The same transducers were also used in the active mode to exploit ultrasonic wave inspection on the same infrastructures.

A group from the University of Arizona (USA) and the Bengal Engineering and Science University (India) proposed a generalized iterative least-squares extended Kalman filter with unknown input system for the health monitoring of frame structures. The methodology is based on a finiteelement-based time-domain system identification technique. It can assess structural health at the element level using only limited number of noise-contaminated responses. The algorithm was verified on simple structural models in which the structure is excited by multiple loadings simultaneously, and it was damaged through various stages of degradation in single or multiple members.

The monitoring of pipes with guided ultrasonic waves and digital processing based on the wavelet transform and the Fourier transform was demonstrated by researchers at the University of Connecticut (USA) and the University of Arizona. The pipes were subjected to various boundary conditions to evaluate the effect of water flow and soil, for instance, on the response of the guided waves to the presence of defects.

A masonry bridge located in Luxembourg, namely, the Adolphe Bridge in Luxembourg City, was monitored for few months using an array of low-cost wireless sensors. The data enabled the estimation of the fundamental frequency of vibration and the mode shape of the bridge. The experiment showed that a network of wireless sensing units can be used to monitor the vibration characteristics of historical arch bridges for SHM applications.

Finally, a probabilistic SHM method to detect global damage in a highway bridge in Connecticut was demonstrated. The bridge is a curved, three-span steel dual-box girder bridge, located in Hartford Connecticut (USA), and it is instrumented with 22 sensors consisting of accelerometers and tilt and temperature sensors. The SHM method described in this special issue accounted for the variability associated with environmental and operational conditions. Three damage measures were identified in this study: the value of fundamental natural frequency determined from 
peak picking of autospectral density functions of the bridge acceleration measurements, the magnitude of the peak acceleration measured during a truck crossing, and the magnitude of the tilt measured at 10-minute intervals.

Piervincenzo Rizzo Lingyu (Lucy) Yu Alessandro Marzani 

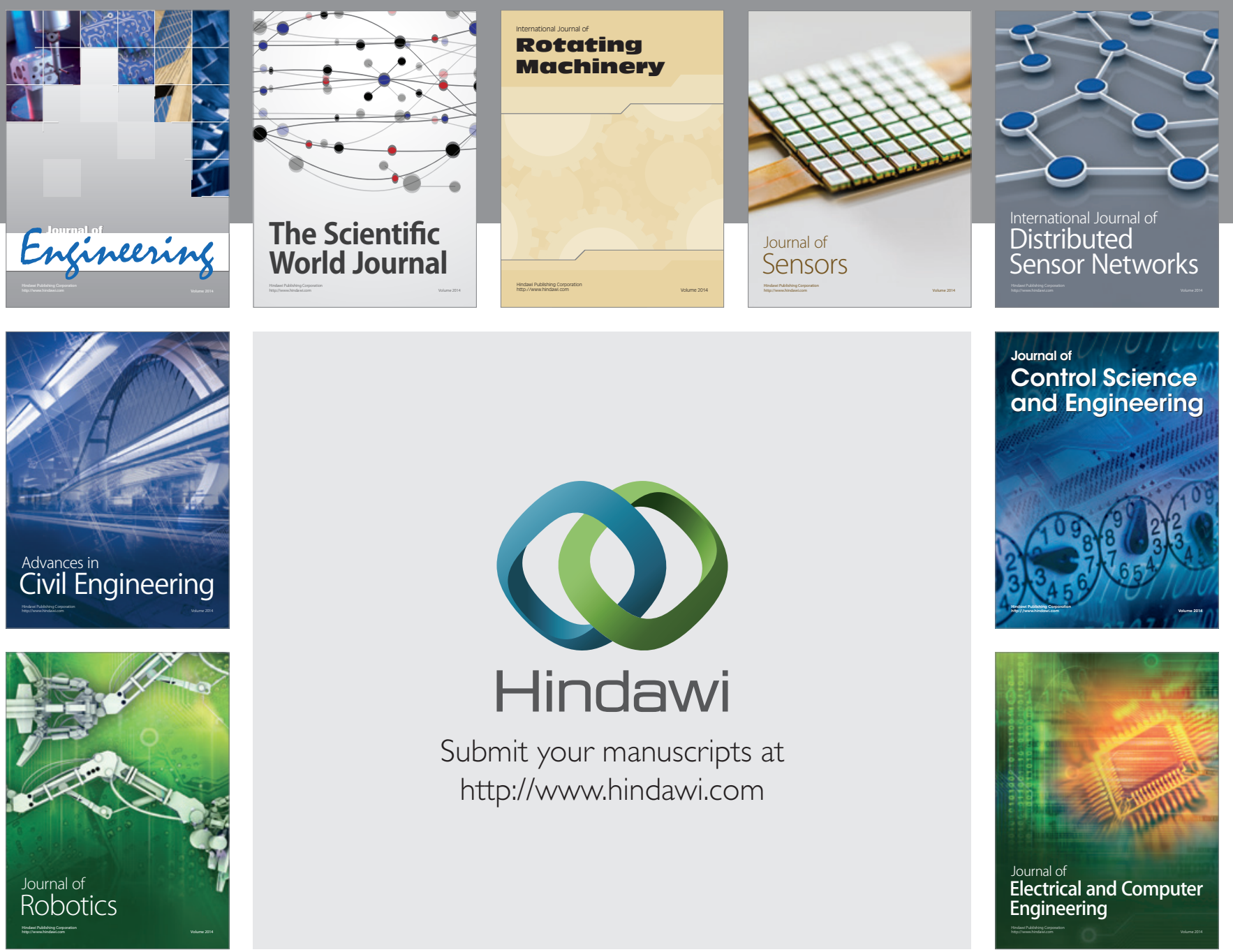

Submit your manuscripts at

http://www.hindawi.com
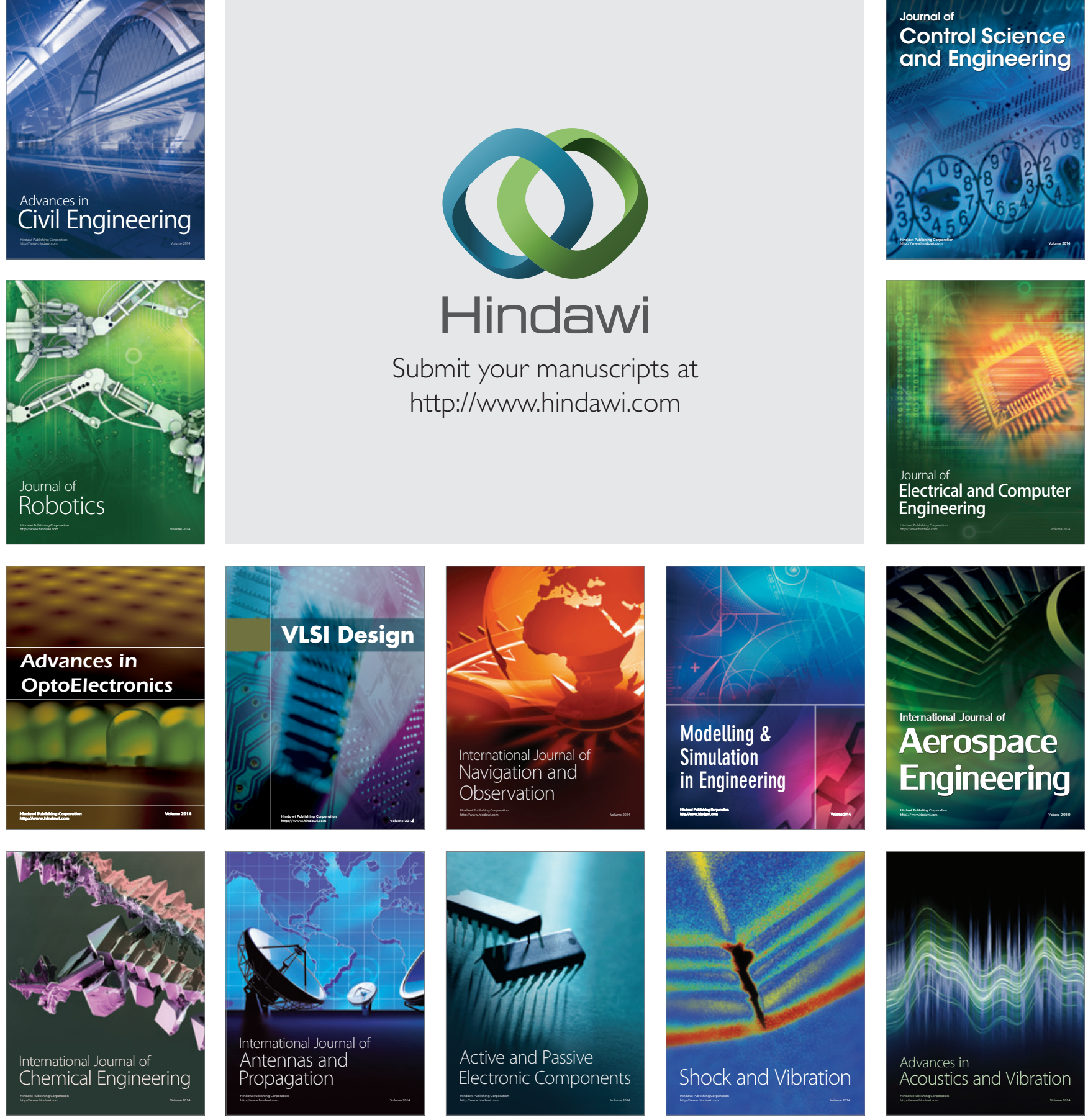